\title{
Improving the efficiency of circular irrigation machines based on models of neural network irrigation control
}

\author{
Galina Kamyshova, ${ }^{1, *}$ Dmitrii Solovyev ${ }^{1}$, Sergei Zatinatsky ${ }^{1}$, Dmitrii Kolganov $^{1}$, Nadezhda \\ Terekhova ${ }^{1}$ \\ ${ }^{1}$ Saratov State Agrarian University, 1, Theatralnaia pl., 410012, Saratov, Russia
}

\begin{abstract}
The article presents the results of studies of the operational efficiency of circular irrigation machines based on models of neural network irrigation control. Existing irrigation machines are not fully able to realize their advantages in irrigation due to the high degree of energy intensity. Traditional approaches based only on physical modeling of technical processes and relationships often make it difficult to find effective solutions. Intelligent irrigation control is essential for maximum efficiency and productivity. An approach based on a model of data mining is proposed, namely, control of a sprinkler using a neurocontroller. Most irrigation systems use ON / OFF controllers. These controllers cannot give optimal results for different time delays and different system parameters. The proposed controller based on an artificial neural network was created using MATLAB. The main modeling parameters are water pressure and speed. Neurocontrol, leads to the possible implementation of better and more effective management of irrigation machines.
\end{abstract}

\section{Introduction}

Agriculture is the world's largest consumer of fresh water, accounting for more than $70 \%$ of its total consumption. Russia is one of the largest agrarian countries in the world. However, most of Russia's agricultural land is located in risky farming zones in which growing crops without irrigation is inefficient. Therefore, in the arid zone, more than $70 \%$ of the areas occupied under the cultivation of agricultural products are included in the system of territories of irrigated agriculture [1]. According to official data [2], the share of reclaimed land in developed countries of the total area of arable land is $39 \%$ in the USA, $35.7 \%$ in India, $54.8 \%$ in China, and $7.9 \%$ in Russia.

Of great importance for the development of crop production on irrigation is the technical equipment of the irrigation system. According to the data of the Land Reclamation Department of the Russian Federation, the sprinkling equipment fleet consists of approximately $30 \%$ of the first-generation irrigation machines DDA-100M (MA) and DDN-70 (100), 60\% of the second-generation irrigation machines of IM "Fregat" and IKSh "Volzhanka", and only $10 \%$ of the third generation irrigation machines IM "Kuban-LK"

\footnotetext{
*Corresponding author: gkamichova@ mail.ru
} 
and foreign-made machines "Zimmatic", "Pivot", "Rain Star", "Valley" [3, 4]. At the same time, the existing fleet of irrigation equipment consists of $80 \%$ of machines that have served their normative period and for the most part, wide-grip machines of the Frigate type, which are very energy-intensive, represent them. The use of such equipment in irrigation determines the high cost of agricultural products due to the inclusion in operating costs, a significant part of the costs associated with water supply for irrigation.

From the foregoing, it follows that for the operation of the second and third generation of irrigation machines necessary to upgrade these machines with the use of modern irrigation technologies cost-effective and environmentally friendly.

Watering crops in Russia and, in particular, in the Saratov region is carried out mainly by sprinkling. At the same time, the main irrigation machine is the Frigate irrigation machines, which has worked well for 40 years of operation on irrigation systems in the Middle and Lower Volga. It provides good rain quality, automation of irrigation, high reliability, and allows you to use one type of energy to provide watering and movement of the machine.

However, as the practice of operation shows, this machine is not fully able to realize its advantages in irrigation due to the high degree of energy intensity. Therefore, for the effective operation of the IM "Frigate", it is necessary to improve the machine by developing new structural elements and control systems.

There are obvious trends in the development of sprinkler technology in the direction of automation and robotization, that is, because of saving energy, water and other resources. Among foreign technology, examples of "Zimmatic", "Valley", "Reinke" can be given.

Russian scientists, including SSAU scientists, are conducting research in the direction of improving the design parameters of sprinkler machines that reduce energy consumption while maintaining the technological parameters of irrigation [5], [6], [7].

As you know, the sprinkler is a complex hydro-, electro, mechanical dynamic system with, often, non-linear dependencies. Therefore, traditional approaches based only on physical modeling of technical processes and relationships often make it difficult to find effective solutions. On the other hand, technological advances that stimulate the exponential growth of data can increase the efficiency of engineering solutions. The quick extraction of relevant information from the modern data stream is now a requirement for achieving results. New tools for data mining, deep learning, etc. provide improved accuracy by resolving complex relationships in large volumes of technical parameters and have great potential.

In recent years, many studies have focused on the application of data mining and deep learning methods to improve irrigation efficiency. Therefore, McCarthy [8] developed a cotton irrigation management system, Navarro-Hel [9], Giusti [10] decision support systems for irrigation management. The present study focuses on increasing the efficiency of sprinkling equipment based on models of neural network irrigation control.

\section{Materials and Methods}

\subsection{Problem statement}

Among the key characteristics of sprinkler machines are the irrigation rate and average rain intensity, which must comply with agricultural requirements. These characteristics depend on many design and operational parameters, the influence of which is not equivalent. However, one of the main ones can be distinguished - the water pressure at the inlet. This factor, in turn, is not independent and affects such characteristics of the sprinkler as energy consumption. 
It was found, that the Frigate irrigation machine is highly energy-intensive and can irrigate in accordance with the norms and requirements of irrigation at pressures in the range $4.5 \ldots 7 \mathrm{~atm}$. At a working pressure of $7 \mathrm{~atm}$, the energy consumption will be 0.190 $\mathrm{kW} \cdot \mathrm{h}$, with a decrease in the working pressure, the required energy consumption will decrease in direct proportion and at a pressure of $4.5 \mathrm{~atm}$ will be $0.115 \mathrm{~kW} \cdot \mathrm{h}$. However, a further decrease in pressure leads to inefficient irrigation, as the irrigation rate issued does not correspond to the recommended values of the norms and timing of irrigation (table. 1).

Table 1. The dependence of the irrigation rate on the inlet water pressure (irrigation system "Frigate").

\begin{tabular}{|c|c|}
\hline $\begin{array}{c}\text { Inlet water pressure, } \\
\text { ATM }\end{array}$ & Irrigation rate, $\mathbf{m}^{\wedge} \mathbf{3}$ /hectare \\
\hline 4 & 200 \\
\hline 5 & 510 \\
\hline 6 & 620 \\
\hline 7 & 905 \\
\hline
\end{tabular}

To solve this problem, it was proposed to use hydraulic modeling [11], on the basis of which hydraulically dependent design parameters were determined. This allowed the modification of the base sprinkler to a low-pressure irrigation machine $[12,13]$.

However, the problem of increasing the efficiency of irrigation machines is still relevant, due to the complexity of the physical modeling of dynamic multicomponent systems with non-linear dependencies and the need to take into account external stochastic factors affecting the process. In addition, the digital transformation of agriculture, which involves the robotization of various processes, including irrigation, determines the need for research to improve systems and algorithms for controlling sprinkler machines, including those implemented based on neurocontrollers.

\subsection{Materials}

The process of controlling the value of the irrigation norm depending on the water pressure at the inlet and the speed of the unit is the object of the study.

Field experiments on data collection were carried out in the Engels district of the Saratov region on the Gagarin irrigation system. According to agro climatic conditions, the study area belongs to the zone of insufficient and unstable humidification, the climate is temperate continental with a predominant number of clear and low cloudy days in a year, hot and dry summers, cold and not very snowy winters, short spring and short autumn, high probability of spring and autumn frosts . High temperatures are in June, July and August, the average maximum in July is $+30^{\circ} \mathrm{C}$, low in January and February, and the average minimum in February is $23^{\circ}$ C. Spring is unstable, cold lasts until April, snow cover reaches $30-40 \mathrm{~cm}$. Summer is hot, droughts are possible, as well as a sharp cooling at the beginning and end. The maximum rainfall of $49 \mathrm{~mm}$ falls in November, the minimum rainfall of $25 \mathrm{~mm}$ in April, rainfall is distributed unevenly. Relative humidity is maximum in winter and reaches $85 \%$, the minimum value in summer is $55 \%$.

The pumping station includes: 2 pumps brand ND 1250, flow rate $-1250 \mathrm{~m} 3 / \mathrm{h}$ with a capacity of $650 \mathrm{~kW} / \mathrm{h}$; working pressure - 1.2 MPa and 2 pumps of the SPNS 100/100 brand, flow rate $-100 \mathrm{~m} 3 / \mathrm{h}$ with a power of $160 \mathrm{~kW} / \mathrm{h}$; working pressure - 1.2 MPa. Watering is carried out from the hydrant of a closed irrigation network with water intake from the irrigation canal. Diagram of a network of irrigation machines with a control unit is presented to Figure 1. 


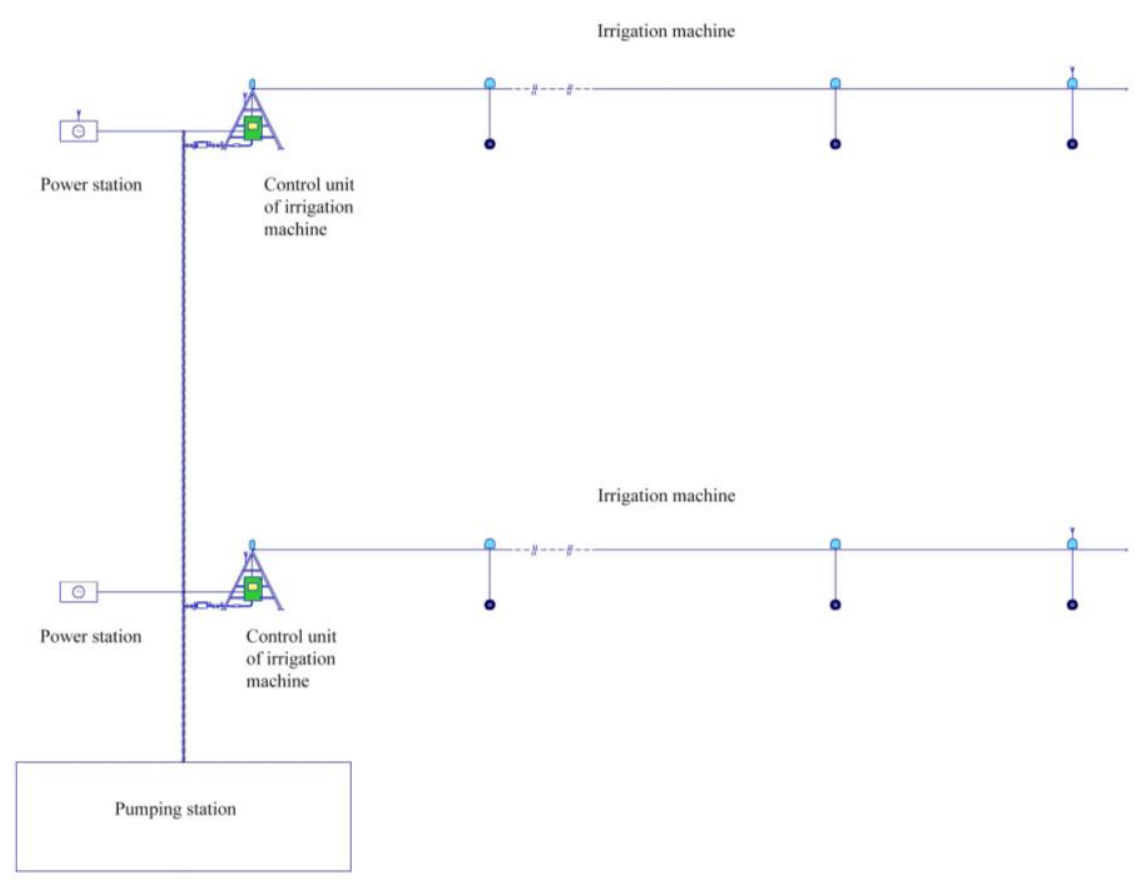

Fig. 1. Diagram of a network of irrigation machines with a control unit.

The graphs of selected data sets of field experiments is presented to Figure 2.

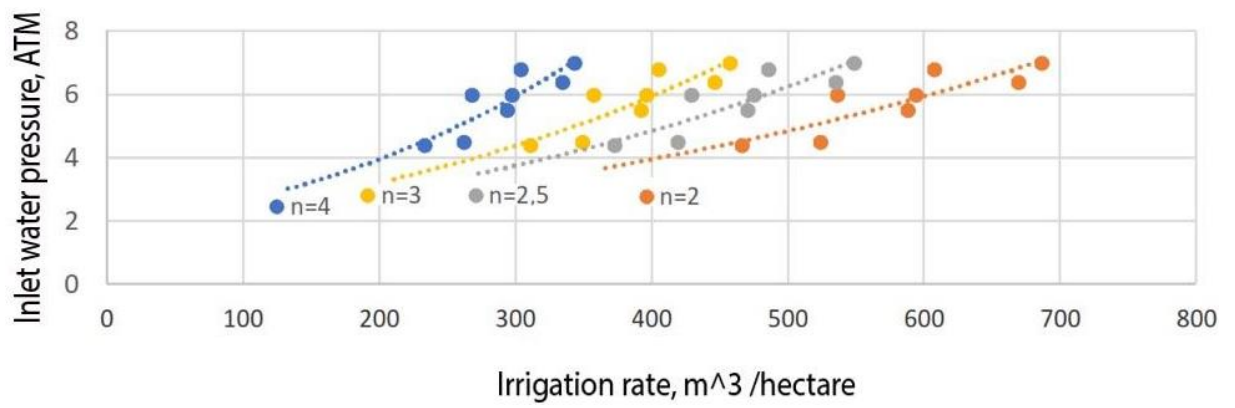

Fig. 2. The graphs of quantities of irrigation rate (irrigation system "Frigate") depending on inlet water pressure and the speed (n).

\subsection{The mathematical description of the method}

Neurocontrol of dynamic objects is a promising new area at the junction of such disciplines as automatic control, artificial intelligence, neurophysiology [14]. The literature describes numerous examples of the practical application of neural networks $(\mathrm{NN})$ for solving tasks of controlling various objects $[15,16]$.

The main element of a neural network is a neuron. An artificial neuron is the simplest analog transforming element that models the basic ideas about the work of a living neuron. Artificial neural networks are parallel computing devices consisting of many interacting simple processors (neurons).

The input of an artificial neuron receives a certain set of signals. Each input is weighted - multiplied by a certain coefficient (synaptic power). The output signal of the adder is fed 
to the input of the activation non-linear block. Usually, activation blocks limit the output of a neuron in a given range, for example, $[0 ; 1]$ or $[-1 ; 1]$.

The mathematical formalization of an artificial neuron is the adder equation

$$
s_{k}=\sum_{j=1}^{n} w_{j, k} \cdot x_{j}+b_{k}
$$

and activation block equation

$$
y_{k}=\phi\left(s_{k}\right)
$$

where, for $k$-th neuron, $x_{1}, x_{2}, \ldots, x_{n}$ - input signals; $w_{1, k}, w_{2, k}, \ldots, w_{n, k}$ - synoptic weights; $b_{k}$-reference signal level; $s_{k}$ - linear adder output; $\phi\left(s_{k}\right)$ - activation block conversion function; $y_{k}$ - output signal. In Fig. 3 presented functional diagram of the artificial neuron model.

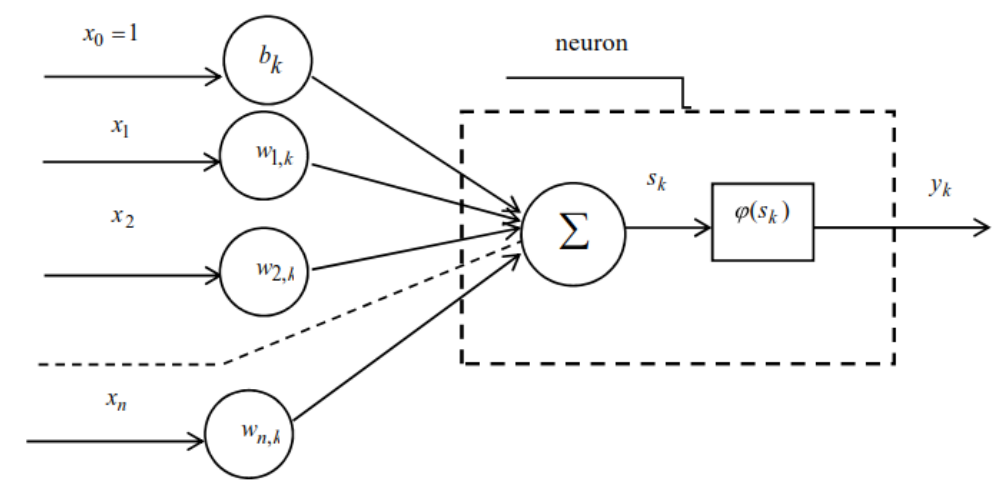

Fig. 3. Functional diagram of the artificial neuron model.

A plurality of associated inputs and outputs of the neurons of the artificial neural network. The principle of operation of the simplest neural network (Figure 3 ) is as follows: ANN perceives the vector of input signals $x=\left(x_{1}, x_{2}, \ldots, x_{n}\right)$ and forms a signal $z=$ $\left(z_{1}, z_{2}, \ldots, z_{m}\right)$ on hidden layer neurons. Neural network outputs vector $y=$ $\left(y_{1}, y_{2}, \ldots, y_{m}\right)$ removed from the output layer. When working with the network, there are two fundamentally different modes - training and work. When training a neural network, a certain training vector of input signals is fed to its input, and the values of the connection weights $w_{i}$ and the shifts $w_{0}$ of the neurons of the hidden and output layers vary until the network learns to map some set of $\mathrm{N}$ training input vectors to the set of desired outputs.

The basis of the neural network is the architecture of the links and the weights corresponding to these links. The communications architecture actually determines how a network is formed from individual neurons. There are no special requirements for the formation of the network architecture; however, there are several standard architectures, of which it is especially convenient to implement in practice:

- direct distribution networks in which graphs do not have loops;

- dynamic (recurrent) networks, or networks with feedbacks.

The simplest network has a direct data transfer structure: data passes from inputs through hidden elements and ultimately comes to output elements. Such a structure has a stable behavior. This is the simplest kind of neural network. Direct distribution networks can be single-layer and multi-layer. 


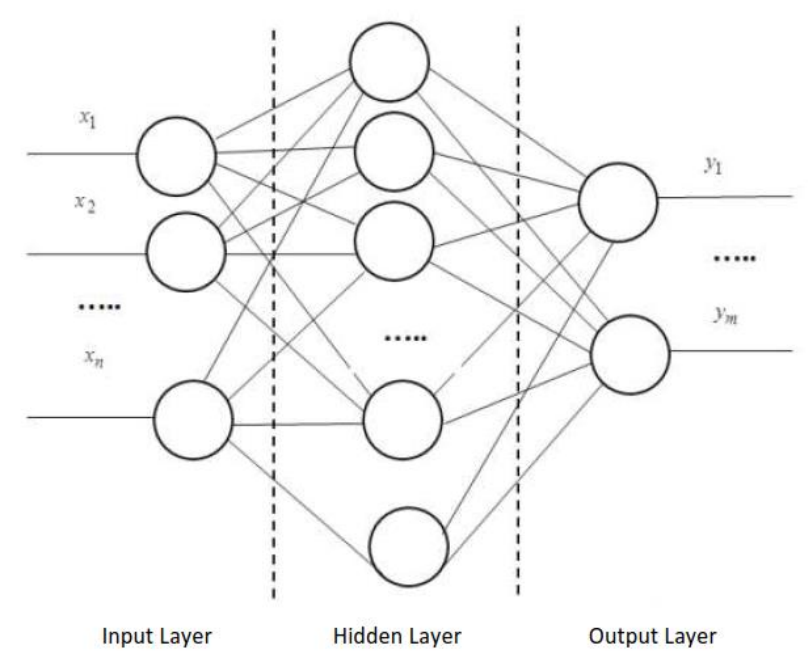

Fig. 4. Functional diagram of a multilayer neural network.

Multilayer neural networks (Figure 4) can be used to identify and control irrigation systems. The neural network acts as an adaptive regulator in the control system of sprinkler machines. In the learning process, an optimal control action is formed, which is fed to the input of the system. The main properties that determine the use of multilayer neural networks for tasks of controlling the characteristics of sprinkler machines:

- approximation properties allowing to implement non-linear control systems;

- adaptive properties, resulting from the ability of neural networks to learn, which allow you to create adaptive control systems;

- Ability to parallel signal processing, allowing to implement control algorithms for multidimensional objects.

The type and structure of the neural network are determined depending on the task. There are optimal configurations for some tasks, as well as recommendations for choosing the type of neural network. If the problem cannot be brought to a known one, a synthesis of a new configuration is required.

Many neural network control schemes have been developed. There are direct methods in which neural networks are used directly to control the process, and indirect methods in which neural networks are used for correction, identification, or filtering. In [17], reviews of control methods using neural networks are presented, as well as algorithms for selecting control quality criteria.

At the first stage, a neuromodel of the control object is compiled, for which in this study we will take the control unit for water pressure and speed. Based on data on water pressure and velocity (u), an object's neuromodel is built and trained, the output signals of which will be as close as possible to the output signals of a real object, which will be the irrigation norm (y).

The training scheme (Figure 5) is constructed as follows: to create a learning set, a random process $\mathrm{u}$ is fed to the input of the control object. The training set is formed from the input control actions $\mathrm{u}$ and the output reactions $\mathrm{y}$. The output signal of the object $\mathrm{y}$ is the irrigation norm, the output signal of the neuromodel is the estimate of the irrigation norm $\hat{y}$. The difference between these values is an error e, which is passed through the neural network in the opposite direction and is used to adjust its weight coefficients. 


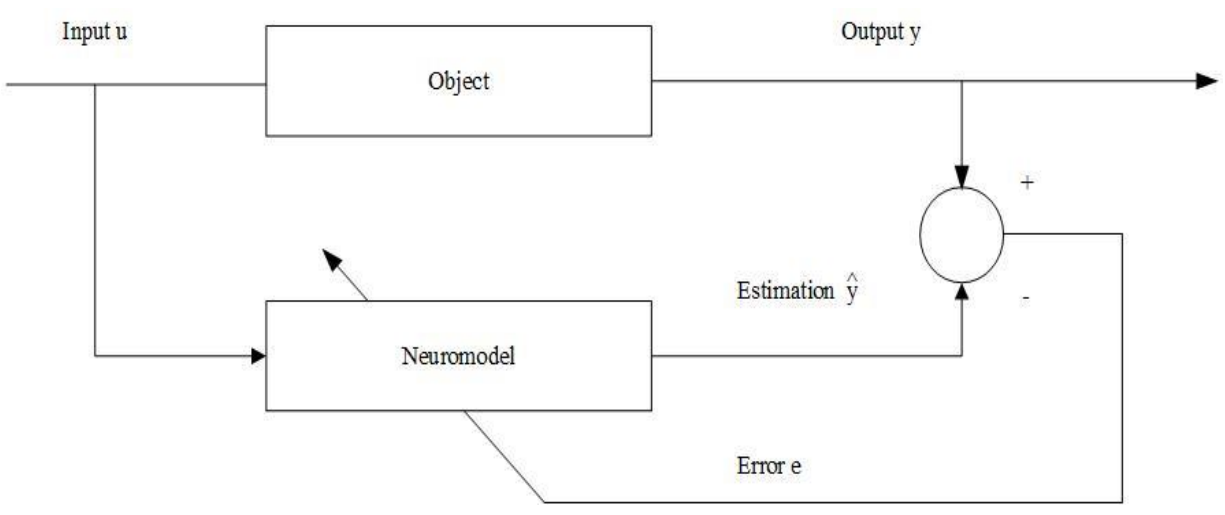

Fig. 5. Neuromodel training scheme.

Then, using the object's neuromodel, the synthesis of the neurocontroller is performed. An inverse neuroemulator acts as a controller [18]. The inverse neurocontrol diagram is shown in Figure 6.

Control

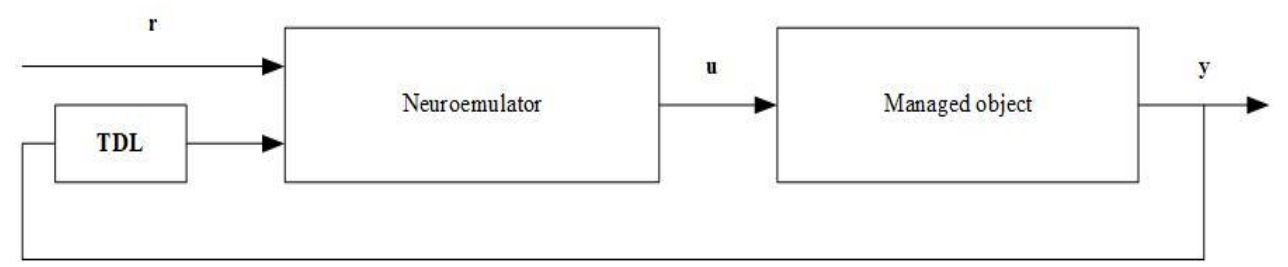

Fig. 6. Object control mode diagram.

In the control process, the reference signal $r$ and the delayed TDL (tapped delay line) signals from the object output are fed to the input of the trained neural network, and the neural network outputs a control signal at the output.

The Neural Network Toolbox of the Matlab system implements a neurocontroller [19] operating according to the algorithm described above. The task of training a neural network is to find the values of $\mathrm{W} 1, \mathrm{~W} 2$ and $\mathrm{b}$, having a data set of $\mathrm{x}$ and $\mathrm{y}$. For this, an iterative learning algorithm will be applied. A neural network is trained based on this data using the Levenberg-Marquardt algorithm. This algorithm most often shows better results than other algorithms, although it requires more memory. The neural network structure was selected (Figure 7), which allows to most accurately describe the dynamics of the system: the number of layers is -2 , the activation function is the hyperbolic tangent, and the quality of learning function is the standard error. The learning process in the Matlab environment is shown in Figure 8.

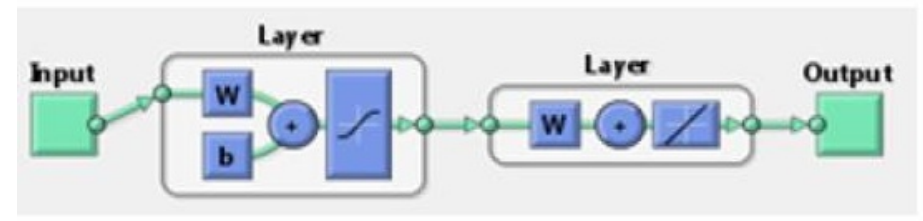

Fig. 7. Neural network structure in Matlab. 


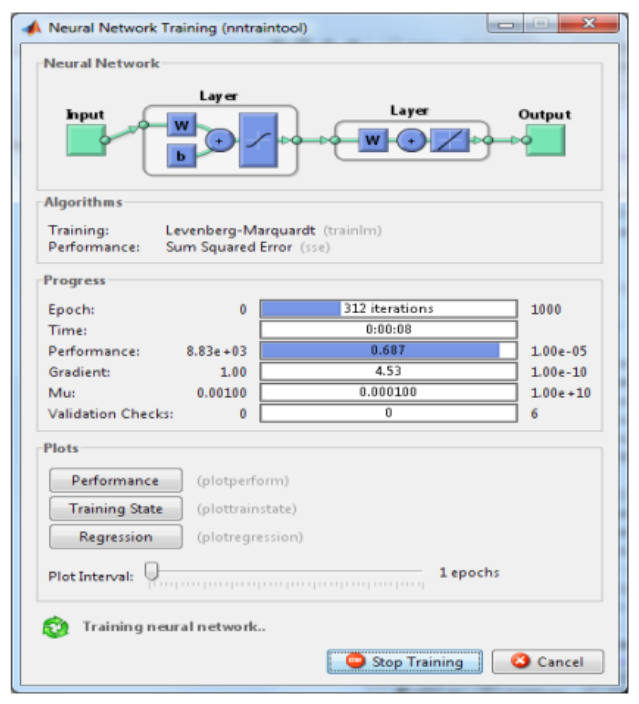

Fig. 8. Training neuromodels in the Matlab.

\section{Results}

As a result of the training of a neural network, its parameters were tuned to the data of field studies, namely, the matrix of weight coefficients of the first layer $W_{1}$, the vector of weight coefficients of the second layer $W_{2}$ and the displacement vector. These parameters determine the neural network, which is the neuromodel of the object.

$$
\begin{aligned}
& W_{1}=\left[\begin{array}{cc}
4.0126 & 6.9171 \\
0.2819 & -0.3232 \\
-0.2703 & 0.2525 \\
-2.0271 & 4.8234
\end{array}\right], W_{2}=\left[\begin{array}{llll}
2.4872 & -0.1347 & -0.3164 & 0.8104
\end{array}\right] \\
& b=\left[\begin{array}{c}
9.7891 \\
48.2249 \\
30.0254 \\
-3.9345
\end{array}\right]
\end{aligned}
$$

After training the neural network, it can be used as a direct controller to control the pressure and speed of the sprinkler. The task of the neurocontroller is to maximally approximate the actual value of the irrigation norm to the required one and optimize such resources as water and energy. 


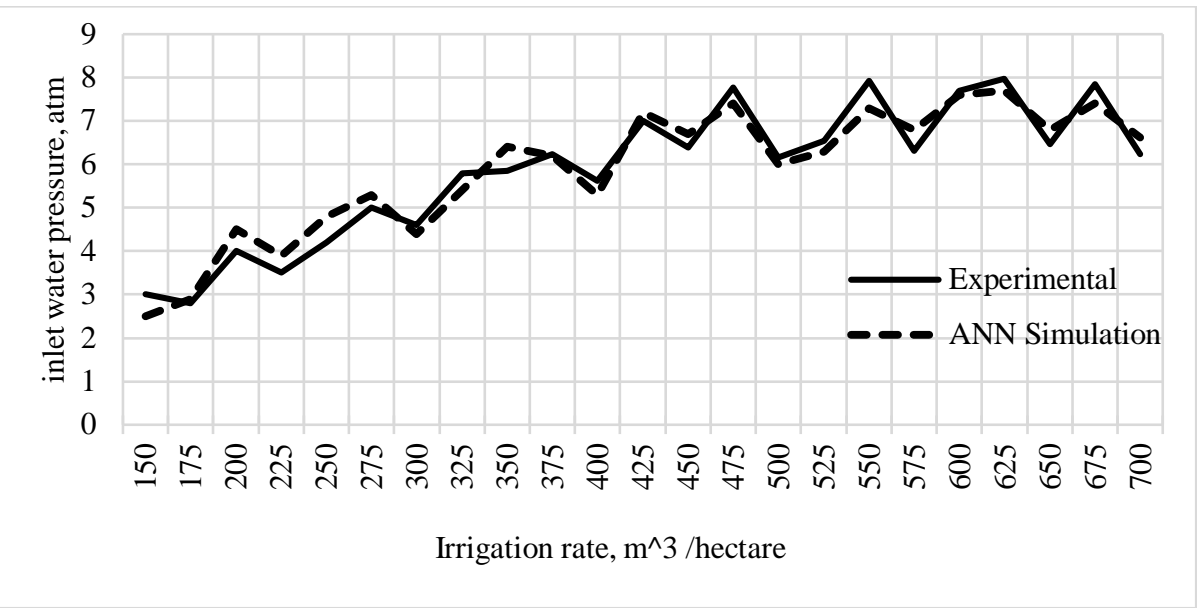

Fig. 9. Simulation results.

A trained neurocontroller is analyzed using MATLAB. The results of application and modeling are shown in Figure 8. As can be seen from the figure, the neural network, which is the model of the controller, correctly generates the output signal on the training set. To verify the adequacy of the model, it is necessary to simulate the output signal of the system on a test set. Based on the results of modeling on a test set, it can be concluded that the neuromodel generates an output signal corresponding to the expected signal, while the error (the difference between the required and the actual value of the irrigation rate) is moderate and reasonable (less than 2\%) is minimal. A neural network is able to convey the dynamics of the process.

\section{Conclusions}

The main goal of developing a cost-effective and result-oriented irrigation control system was achieved by modeling a neurocontroller. This article describes a simple approach to the problem of sprinkler control using an artificial neural network controller. Comparison of the proposed model with typical control models has several advantages.

An approach based on neurocontrol leads to the possible implementation of better and more effective management. Neurocontrollers do not require prior knowledge of the system and have the ability to adapt to changing conditions, unlike traditional methods. This optimizes the irrigation parameters and reduces the cost of electricity and water by 10 $15 \%$, with a sprinkler machine with a neurocontroller in the control system.

\section{References}

1. G. Olgarenko, Land Reclamation and Water Management 1, 44-47 (2018)

2. G. Balakai, Scientific journal of the Russian Research Institute for Land Reclamation 2, 8-10 (2011)

3. V. Shchedrin, A. Kolganov, Irrigation systems of Russia: from generation to generation (2013)

4. Technical level of domestic and foreign equipment used in land reclamation: Information collection (2011)

5. N. Koshkin, Land Reclamation and Water Management 4, 22-25 (2014) 
6. N. Koshkin, D. Soloviev, S. Zatinatsky, Land Reclamation and Water Management 4, 23-26 (2015)

7. B. Fokin, A. Nosov, Modern problems of using multi-support sprinkling machines (2011)

8. A. McCarthy, N. Hancock, S. Raine, Comput. Electron. Agric 101, 135-147 (2014) doi: 10.1016/j.compag.2013.12.004

9. H. Navaro-Hellin, J. Martinez-del-Ricon, R. Domingo-Miguel, F. Soto-Valles, R. Torres-Sanchez, Comput. Electron. Agric 124, 121-131 (2016) doi: 10.1016/j.compag.2016.04.003

10. E. Giusti, S. Marsili -Libelli, Environ. Model. Softw. 63, 73-86 (2015) doi: 10.1016/j.envsoft.2014.09.020

11. F. Abdrazakov, S. Zatinatsky, A. Povarov, International Journal of Pharmacy \& Technology 8, 27063-27071 (2016)

12. D. Soloviev, D. Kolganov, M. Zagoruyko, M. Eliseev, Agrarian Scientific Journal 2, 67-69 (2017)

13. S. Zatinatsky, D. Kolganov, M. Zagoruyko, Agrarian Scientific Journal 7, $69-72$ (2017)

14. A. Chernodub, D. Dziuba, Problems of programming 2, 79-94 (2011)

15. V. Meshcheryakov, P. Saraev, O. Meshcheryakova, Management Problems 6, 71-73 (2013)

16. M. Belov, I. Nosirov, C. Fyong, Izvestia SPbGETU "LETI” 4, 73-80 (2018)

17. J. Schmidhuber, Neural Network 61, 85-117 (2015)

18. K. Cheon, J. Kim, M. Hamadache, D. Lee, J. Autom. Control Eng. 3, 452-456 (2015)

19. V. Dyakonov, MATLAB. Complete tutorial (2012) 